\title{
On a Recursion Formula and on Some Tauberian Theorems
}

\author{
N. G. de Bruijn ${ }^{2}$ and P. Erdös ${ }^{3}$
}

\begin{abstract}
The paper is concerned with two sets of positive numbers, $c_{k}$ and $f_{k}$, connected by a linear recursion formula. Under certain assumptions there exists an asymptotic relation between the partial sums $\sum_{1}^{n} c_{k}$ and $\sum_{1}^{n} f_{k}$.

The assumptions on the $c_{k}$ are of Tauberian type. The method is based on discussing the associated power series $\sum_{1}^{\infty} c_{k} x^{k}$ and $\sum_{1}^{\infty} f_{k} x^{k}$.
\end{abstract}

Let

Define

$$
c_{k} \geq 0, \quad \sum_{k=1}^{\infty} c_{k}=1
$$

$$
f(1)=1, \quad f(n)=\sum_{k=1}^{n-1} c_{k} f(n-k) \quad(n>1) .
$$

This recursion formula has various applications in the theory of probability. ${ }^{4}$ In the present note, however, we will investigate (1) independently of its applications. Assume, first, that

$$
\sum_{k=1}^{\infty} k c_{k}<\infty
$$

Erdös, Feller, and Pollard [2] proved that if the greatest common divisor of the $k$ 's with $c_{k}>0$ is 1 , then,

$$
f(n) \rightarrow A^{-1} \quad\left(A=\sum_{k=1}^{\infty} k c_{k}\right) .
$$

It is easy to see that if the greatest common divisor of the $k$ 's with $c_{k}>0$ is greater than 1 , then $\lim f(n)$ cannot exist. ${ }^{5}$ It was also shown that if

$$
\sum_{1}^{\infty} k c_{k}=\infty,
$$

then (2) always holds, in other words, $f(n) \rightarrow 0$.

Feller in a paper [3] restricted himself to the case when $\sum k c_{k}<\infty$. In the present paper we will not in general make this assumption.

We prove the following results:

Theorem 1. Assume that for every $k>1$,

$$
c_{k-1} c_{k+1}>c_{k}^{2} \text {. }
$$

Then for every $n>1$,

$$
f(n-1) f(n+1)>f^{2}(n) .
$$

${ }^{1}$ Preparation of this paper was sponsored in part by the Office of Naval Research.

${ }_{2}^{2}$ Technische Hogeschool Delft. Present address: University of Amsterdam, Amsterdam, Holland.

${ }^{3}$ National Bureau of Standards, and University of Aberdeen, Scotland. 4 See Feller [3]. This paper quotes most of the literature that deals with these cuestions.

${ }_{5}^{5}$ See [2] and the remarks at the end of the present paper.
Other theorems of the same type as theorem 1 were proved by T. Kaluza [4]. Assuming (1), he showed for instance, that $f(2)>0, f(n-1) f(n+1)>f^{2}(n)$ $(n=2,3, \ldots)$ imply that the $c$ 's are positive. Furthermore, he proved that $f(1), f(2), \ldots$ is a moment sequence if, and only if, $c_{1}, c_{2}, c_{3}, \ldots$ is a moment sequence. (Here $c_{1}, c_{2}, c_{3}, \ldots$ is called a moment sequence whenever it is of the form $\alpha_{n}=\int_{0}^{\infty} u^{n} d \chi(u)$, where $\chi(u)$ is nondecreasing and such that the integral converges for all $n$ ).

Theorem 2. Putr $r_{k}=\sum_{l \geq k} c_{l}, s(y)=\sum_{k \leq y} r_{k}, S(y)=\sum_{k \leq y} f(k)$. Assume that for every $p>0$

$$
\lim _{y \rightarrow \infty} \frac{s(p y)}{s(y)}=p^{\alpha}
$$

for a fixed $\alpha, 0 \leq \alpha \leq 1$ ( $\alpha$ independent of $p$ ). Then

$$
s(y) S(y)=\frac{y}{\Gamma(2-\alpha) \Gamma(1+\alpha)}+o(y) .
$$

Theorem 3. Assume that (3) and (4) both hold. Then,

$$
f(n)=\frac{1-\alpha}{s_{n} \Gamma(1+\alpha) \Gamma(2-\alpha)}+o\left(\frac{1}{s_{n}}\right)
$$

In case $\alpha=1,(6)$ does not give an asymptotic formula, it only gives $f(n)=o\left(s_{n}{ }^{-1}\right)$.

It would be interesting to obtain conditions that imply $f(n+1) / f(n) \rightarrow 1$. We can prove that if $c_{n+1} / c_{n} \rightarrow 1$, then $f(n+1) / f(n) \rightarrow 1$; also if

$$
c_{n}<B . \min _{1 \leq k \leq n} c_{k}
$$

then $f(n+1) / f(n) \rightarrow 1$. We suppress the proofs because we believe that very much more general conditions can be obtained. If $f(n+1) / f(n) \rightarrow 1$, then it is not difficult to prove that $c_{n-1}=o\{f(n)\}$. It can be conjectured that the converse is also true, under the additional condition that the g.c.d of the $k$ 's with $c_{k}>0$ is 1 . 
Proof of theorem 1. First we show that for any $n$ $c_{n}\left\{f(n+2) f(n)-f^{2}(n+1)\right\}$

$$
\begin{aligned}
=\sum_{k=2}^{n}\left(c_{n+1} c_{k-1}-c_{n} c_{k}\right)\{f(n+1) f(n+1-k) \\
-f(n) f(n+2-k)\} .
\end{aligned}
$$

To prove (7) split the right-hand side into four sums. These are, respectively,

$$
\begin{aligned}
c_{n+1} f(n+1) \sum_{k=2}^{n} c_{k-1} f(n+1-k)=c_{n+1} f(n+1) f(n) & \\
-c_{n+1} f(n) \sum_{k=2}^{n} c_{k-1} f(n+2 & -k) \\
& =-c_{n+1} f(n)\left\{f(n+1)-c_{n} f(1)\right\} ; \\
-c_{n} f(n+1) \sum_{k=2}^{n} c_{k} f(n+ & 1-k) \\
& =-c_{n} f(n+1)\left\{f(n+1)-c_{1} f(n)\right\} ;
\end{aligned}
$$

$c_{n} f(n) \sum_{k=2}^{n} c_{k} f(n+2-k)$

$$
=c_{n} f(n)\left\{f(n+2)-c_{n+1} f(1)-c_{1} f(n+1)\right\} .
$$

Addition gives $c_{n}\left\{f(n+2) f(n)-f^{2}(n+1)\right\}$, which proves (7).

To prove theorem 1, observe that

$$
f(1) f(3)-f^{2}(2)=c_{1} f(2)+c_{2} f(1)-f^{2}(2)=c_{2} f(1)>0 \text {. }
$$

((3) implies that all the c's are positive.) Assume now $n>2$, and suppose that $f(k) f(k+2)>f^{2}(k+1)$ is already proved for $1 \leq k<n$. Then (3) implies $c_{n+1} c_{k-1}>c_{n} c_{k}$, since by $(3)\left(c_{2} / c_{1}\right)<\left(c_{3} / c_{2}\right)<\ldots$. Thus in (7) all terms on the right side are positive, and we obtain $f(n) f(n+2)>f^{2}(n+1)$, which proves theorem 1.

Remarks: It is clear from the proof of theorem 1 that if we only assume that $c_{k+1} c_{k-1} \geq c_{k}^{2}(k>1)$, we obtain $f(n+1) f(n-1) \geq f^{2}(n)(n>1)$.

If (3) is true, then, by theorem $1, f(n+1) / f(n)$ is an increasing function of $n$. We have $f(n+1) / f(n)<1$ for all $n$, for otherwise we would have $f(n+1)$ $f(n)>a>1$ for some $a$ and all large $n$. This would contradict the fact that $f(n)=O(1)$, which easily follows from (1). From $f(n+1)<f(n)(n=1,2, \ldots)$ it follows that

$$
f(n)\left(c_{1}+\ldots+c_{n}\right)<f(n+1)<f(n),
$$

and so (3) implies $f(n+1) / f(n) \rightarrow 1 \quad(n \rightarrow \infty)$.

To prove theorem 2 we need some lemmas.

Lemma $1 .{ }^{6}$ Let $d_{1}, d_{2}, \ldots$ be an infinite sequence, and let $\alpha$ be a number greater than -1 . Put $g(y)=$ $\sum d_{k}$, and assume that $g(y)>0$ for all large $y$, and that,

As far as the authors know, a complete proof of this lemma was not published before, although it is the Abelian counterpart of the Tauberian lemma 2, which is due to Karamata. K. L. Chung brought to our notice that in Doetsch [1] an incomplete proof is presented for a theorem very similar to our lemma 1. Doetsch claims to use only the inequalities $L(y)=O\left(y^{\epsilon}\right), 1 / L(y)=O\left(y^{\epsilon}\right)(y \rightarrow \infty)$, whereas an inequality of the type (11) seems to be indispensable. for every $p>0$,

$$
g(p y) / g(y) \rightarrow p^{\alpha} \quad(y \rightarrow \infty) .
$$

Then the series $D(x)=\sum_{1}^{\infty} d_{k} x^{k}$ converges for $|x|<1$, and if $t>0, t \rightarrow 0$, we have

$$
D\left(e^{-t}\right)=\{1+o(1)\} g(1 / t) \Gamma(1+\alpha) .
$$

Proof. The function $L(y)=g(y) y^{-\alpha}$ is positive for $y$ large, and it is measurable and bounded over any finite interval $0 \leq y \leq A$ (for $g(y)=0$ if $0 \leq y<1$ ). Furthermore, $L(y)$ is slowly increasing, that is, $L(p y) / L(y) \rightarrow 1$ as $y \rightarrow \infty$, for every $p>0$.

We shall prove that for any $\epsilon>0$ there exist positive constants $C(\epsilon), C_{1}(\epsilon)$ such that

$$
\left|\frac{L(p y)}{L}(y)\right|<C_{1}(\epsilon)\left\{p^{\epsilon}+p^{-\epsilon}\right\} \quad(p>0, y>C(\epsilon)) .
$$

It is known ${ }^{7}$ that $\mathrm{L}(p y) / L(y) \rightarrow 1$ as $y \rightarrow \infty$, uniformly for $a \leq p \leq b$, where $a$ and $b$ are arbitrary positive. Therefore, $C(\epsilon)$ can be determined such that $L(y)>0$ for $y \geq C(\epsilon)$ and such that

$$
\log \{L(p y) / L(y)\}<\epsilon \quad\left(e^{-1} \leq p \leq e, y \geq C(\epsilon)\right) .
$$

It follows by induction that

$\log \{L(p y) / L(y)\}<\epsilon(1+\log p) \quad(p \geq 1, y \geq C(\epsilon))$,

and

$\log \{\boldsymbol{L}(p y) / L(y)\}<\epsilon\left(1+\log p^{-1}\right)$

$$
\left(C(\epsilon) y^{-1} \leq p \leq 1, \quad y \geq C(\epsilon)\right) .
$$

Put

$$
M(\epsilon)=\sup _{0 \leq y \leq C(\epsilon)} L(y)
$$

Then we have, for $0<p<C(\epsilon) y^{-1}, \quad y \geq C(\epsilon)$ by (13),

$$
\begin{aligned}
& \log \{L(p y) / L(y)\} \\
& =\log \{L(C(\epsilon)) / L(y)\}+\log \{L(p y) / L(C(\epsilon))\} \\
& <\epsilon\left\{1+\log \frac{y}{C(\epsilon)}\right\}+\log \frac{M(\epsilon)}{L(C(\epsilon))} \\
& <\epsilon\left(1+\log p^{-1}\right)+C_{2}(\epsilon) .
\end{aligned}
$$

Now (12), (13) and (14) prove (11).

In the first place, we obtain from (11) that $L(x)=O\left(x^{\epsilon}\right)$ as $x \rightarrow \infty$, and therefore $d_{k}=O\left(k^{\alpha+\epsilon}\right)$. Hence the power series for $D(x)$ converges if $|x|<1$.

We have, for $t>0$,

$$
D\left(e^{-t}\right)=\int_{0}^{\infty} e^{-y t} d g(y)=\int_{0}^{\infty} t e^{-y t} g(y) d y
$$

7 See [5] (where $L(y)$ is assumed to be continuous), and [7]. 
and so,

$$
D\left(e^{-t}\right)=t^{-\alpha} L\left(t^{-1}\right) \int_{0}^{\infty} \phi(y, t) d y
$$

where

$$
\phi(y, t)=e^{-y} y^{\alpha} \frac{L(y / t)}{L(1 / t)} .
$$

For any fixed $y>0, \phi(y, t)$ tends to $e^{-y} y^{\alpha}$ as $t \rightarrow 0$. Furthermore, by $(11), \phi(y, t)$ can be majorized by a positive function of $y$ only, whose integral over $(0, \infty)$ converges. Therefore, by the Arzéla-Lebesgue theorem, we have

$$
\int_{0}^{\infty} \phi(y, t) d y \rightarrow \int_{0}^{\infty} e^{-y} y^{\alpha} d y=\Gamma(1+\alpha) \quad(t>0, t \rightarrow 0) .
$$

This proves the lemma.

Lemma 2. Assume that

$$
D(x)=\sum_{1}^{\infty} d_{k} x^{k}
$$

is convergent for $|x|<1$, and that $d_{k} \geq 0$ but not all $d_{k}=0$. Let $\alpha \geq 0$ be fixed. Assume that for any fixed $p>0$

$$
D\left(e^{-p t}\right) / D\left(e^{-t}\right) \rightarrow p^{-\alpha} \quad(t>0, t \rightarrow 0) .
$$

Then we have

$$
\sum_{k \leq t-1} d_{k}=\{1+o(1)\} D\left(e^{-t}\right) / \Gamma(1+\alpha) \quad(t>0, t \rightarrow 0) .
$$

This result is due to Karamata [6].

Theorem 2 can be derived from lemmas 1 and 2 . Following a suggestion of Karamata, we first prove a more general theorem:

Theorem 4. Let $a_{k} \geq 0$ (but not all $=0$ ), $b_{k} \geq 0$ (but not all $=0), k=1,2,3, \ldots$;

$$
d_{n}=\sum_{1}^{n-1} a_{k} b_{n-k} \quad(n=2,3, \ldots) .
$$

Put

$$
s(y)=\sum_{k \leq y} a_{k}, \quad S(y)=\sum_{k \leq y} b_{k}, \quad T(y)=\sum_{k \leq y} d_{k} .
$$

Assume that for every $p>0$, we have

$$
s(p y) / s(y) \rightarrow p^{\alpha}, \quad T(p y) / T(y) \rightarrow p^{\gamma} \quad(y \rightarrow \infty),
$$

where $\gamma \geq \alpha \geq 0, \gamma$ and $\alpha$ independent of $p$. Then we have

$$
S(y)=\{1+o(1)\} \frac{T(y)}{s(y)} \frac{\Gamma(1+\gamma)}{\Gamma(1+\gamma-\alpha) \Gamma(1+\alpha)} .
$$

Proof. Put $\mathrm{A}(x)=\sum_{1}^{\infty} a_{k} x^{k}, \quad B(x)=\sum_{1}^{\infty} b_{k} x^{k}$, $\mathrm{D}(x)=\sum_{2}^{\infty} d_{k} x^{k}$, then we have formally $\mathrm{A}(x) B(x)=D(x)$. Both $\mathrm{A}(x)$ and $D(x)$ are analytic for $|x|<1$ (see lemma 1 ); it follows that $B(x)$ is analytic in some circle $|x|<\delta$. The coefficients of $B(x)$ are nonnegative, and for $0 \leq x<1, B(x)$ is analytic (since $A(x)>0$ for $0<x<1$ ). Thus by a theorem of Pringsheim (see [8], sec. 17) $B(x)$ is analytic for $|x|<1$.

By lemma 1 we have, as $t>0, t \rightarrow 0$,

$$
A\left(e^{-t}\right) \sim s\left(t^{-1}\right) \Gamma(1+\alpha) ; \quad D\left(e^{-t}\right) \sim T\left(t^{-1}\right) \Gamma(1+\gamma) .
$$

Hence for any $p>0$,

$$
B\left(e^{-p t}\right) / B\left(e^{-t}\right) \rightarrow p^{-\alpha-\gamma} .
$$

But then by lemma 2

$$
S\left(t^{-1}\right) \sim B\left(e^{-t}\right) / \Gamma(1+\gamma-\alpha) .
$$

Now theorem 4 follows immediately from $D(x)=A(x) B(x)$.

Proof of theorem 2. Theorem 2 is an easy consequence of theorem 4 . If

$F(x)=f(1) x+f(2) x^{2}+\ldots, \quad R(x)=r_{1} x+r_{2} x^{2}+\ldots$,

then it follows from (1) that $F(x) R(x)=x^{2} /(1-x)$, and so

$$
\sum_{k=1}^{n-1} r_{k} f(n-k)=1 \quad(n=2,3, \ldots)
$$

Therefore, taking

$$
\begin{gathered}
a_{k}=r_{k}, \quad b_{k}=f(k) \quad(k=1,2, \ldots), \\
d_{n}=1 \quad(n=2,3, \ldots), \quad \gamma=1,
\end{gathered}
$$

we obtain from theorem 4

$$
S(n) \sim \frac{n}{s(n)} \cdot \frac{\Gamma(2)}{\Gamma(2-\alpha) \Gamma(1+\alpha)},
$$

which proves theorem 2 .

Proof of theorem 3. Let $\epsilon$ be a number greater than 0 . From (8) we infer

$$
f(n)>\left\{S_{n(1+\epsilon)}-S_{n}\right\} /(\epsilon n+1) .
$$

It follows from (4) and (5) that

$$
s_{n} S_{n} \sim C n, \quad s_{n} S_{n(1+\epsilon)} \sim C n(1+\epsilon)^{1-\alpha},
$$

where $C=1 /\{\Gamma(2-\alpha) \Gamma(1+\alpha)\}$. Therefore, (17) implies

$$
\lim \inf f(n) s_{n} \geq C\left\{(1+\epsilon)^{1-\alpha}-1\right\} / \epsilon \quad(n \rightarrow \infty) .
$$

This holds for every $\epsilon>0$. Making $\epsilon \rightarrow 0$, we obtain $\lim \inf f(n) s_{n} \geq(1-\alpha) C$. 
Applying the same argument to $n(1-\epsilon)$ instead of $n(1+\epsilon)$ we obtain $\lim \inf f(n) s_{n} \leq(1-\alpha) C$. This proves theorem 3.

Some final remarks: Feller [3] proved the following theorem: Assume that the g.c.d. of the $k$ 's with $c_{k}>0$ is 1 , and that

$$
\sum_{1}^{\infty} k^{2} c_{k}<\infty
$$

then

$$
\sum_{l=1}^{n} f(l)=A^{-1} n+d+o(1),
$$

where $A=\sum_{1}^{\infty} c_{k}$, and, in fact, $\sum_{1}^{\infty}\left\{f(l)-A^{-1}\right\}<\infty$. Now we show the converse, namely, if (19) holds, then (18) holds too.

Theorem 5. Assume that the g.c.d. of the k's with $c_{k}>0$ is 1 , and that $\sum_{1}^{\infty} k^{2} c_{k}=\infty$. Then we have

$$
\sum_{1}^{\infty}\left\{f(l)-A^{-1}\right\}=\infty \text {. }
$$

Proof. If $A=\infty$, then (19) expresses that $\sum_{1}^{\infty} f(l)<\infty$. This is false, since $\sum_{1}^{\infty} f(l) x^{l}=x /\left\{1-\sum_{1}^{\infty} c_{k} x^{k}\right\}$, and the right-hand side tends to $\infty$ if $x \rightarrow 1$. (16),

Now assume $A<\infty$. Since $f(l) \rightarrow A^{-1}$, we have by

$$
\begin{aligned}
\sum_{1}^{n} f(l) \cdot \sum_{1}^{n} r_{k} & =\sum_{2 \leq k+l \leq n} f(l) r_{k}+\sum_{l=1}^{n} f(l) \sum_{n+1-l}^{n} r_{k} \\
& =n-1+\sum_{l=1}^{n}\left(A^{-1}+\boldsymbol{\epsilon}_{l}\right) \sum_{n+1-l}^{n} r_{k} \\
& =n-1+A^{-1} \sum_{k=1}^{n} k r_{k}+\sum_{l=1}^{n} \epsilon_{l} \sum_{n+1-l}^{n} r_{k} \\
& =n-1+\sum_{1}+\sum_{2} .
\end{aligned}
$$

We have $\sum_{1} \rightarrow \infty$, since $\sum k r_{k}$ diverges $\left(\sum k r_{k}>\frac{1}{3} \sum k^{2} c_{k}\right)$, and $\sum_{2}=o\left(\sum_{1}\right)$, since $\epsilon_{l} \rightarrow 0$.

Finally, we have $\sum_{1}^{\infty} r_{k}=\sum_{1}^{\infty} k c_{k}=A$, and so

$$
A \sum_{1}^{n} f(l)>n+\sum_{1}+o\left(\sum_{1}\right) \text {. }
$$

Consequently,

$$
\sum_{1}^{n}\left\{f(l)-A^{-1}\right\}>\left\{A^{-1}+o(1)\right\} \sum_{1} \rightarrow \infty
$$

q.e.d.
Let $D$ denote the greatest common factor of the $k$ 's with $c_{k}>0$. Erdös, Feller, and Pollard [2] proved that if $D=1$ and $\sum k c_{k}<\infty$, then

$$
\sum_{2}^{\infty}|f(k)-f(k-1)|<\infty,
$$

which, of course, implies that $f(k)$ tends to a limit. It seems possible that the condition $\sum k c_{k}<\infty$ is superfluous.

If $D>1$ and $\sum k c_{k}<\infty$, then (20) does not hold, since $\lim f(k)$ does not exist. In order to see this, take $c_{k}^{*}=c_{k D}, f^{*}(k)=f(k D-D+1)$; it follows that

Hence,

$$
f^{*}(k) \rightarrow\left(\sum k c_{k}^{*}\right)^{-1}=D A^{-1} .
$$

$$
f(k D+1) \rightarrow D A^{-1} \neq 0, \quad f(k D+2) \equiv 0 .
$$

If $D>1$ and $\sum k c_{k}=\infty$, then we have $f(k) \rightarrow 0$. Nevertheless, the series (20) need not converge. Take $c_{n}=0$ for $n$ odd, $c_{n}=24 \pi^{-2} n^{-2}$ for $n$ even. Then we have $f(2 n)=0, f(2 n-1)=f^{*}(n)$, where $f^{*}(n)$ and $c_{n}^{*}=c_{2 n}$ are related by an equation of the type (1), and $\sum_{1}^{\infty} c_{n}^{*}=1$. It follows, by theorem 3 , that $f^{*}(n) \sim \pi^{2} /(6 \log n)$.

Therefore,

$$
f(2 n-1) \sim \pi^{2} /(6 \log n), f(2 n)=0,
$$

and the series (20) diverges.

[1] Doetsch, Theorie und Anwendung der Lapiace-Transformationen (Springer, Leipzig, 1937).

[2] P. Erdös, W. Feller and H. Pollard, A property of power series with positive coefficients, Bul. Am. Math. Soc. 55, 201-204 (1949). See also, Kolmogorov Bul. de l'Université d'Etat à Moscow, Sec. A. 1, 1-15 (1937).

[3] W. Feller, Fluctuation theory of recurrent events, Trans. Am. Math. Soc. 67, 98-119 (1949).

[4] T. Kaluza, Ueber die Koeffizienten reziproker Funktionen, Math. Z. 28, 161-170 (1928).

[5] J. Karamata, Sur un mode de croissance régulière des fonctions, Mathematica (Cluj) 4, 38-53 (1930).

[6] J. Karamata, Neuer Beweis und Verallgemeinerung der Tauberschen Sätze, welche die Laplacesche und Stieltjessche Transformation betreffen, J. Reine Ângew. Math. 164, 27-39 (1931).

[7] J. Korevaar, T. van Aardenne-Ehrenfest and N. G. de Bruijn, A note on slowly oscillating functions, Nieuw. Ảrch. Wiskunde (2) 23, 77-86 (1949).

[8] E. Landau, Darstellung und Begründung einiger neuerer Ergebnisse der Funktionentheorie, (Springer, Berlin, 2e Aufl. 1929).

Los Angeles, August 10, 1950. 